\title{
HIGH BRIGHTNESS, HIGH CURRENT INJECTOR DESIGN FOR THE CORNELL ERL PROTOTYPE ${ }^{\alpha}$
}

\author{
I.V. B azarovy and C.K. Sinclair, Wilson L aboratory, Cornell University, Ithaca, NY 14850
}

\begin{abstract}
Cornell University, in collaboration with Jefferson Laboratory, has proposed the construction of a $100 \mathrm{MeV}, 100$ $m A$ CW Energy Recovery Linac (ERL) prototype, to study and resolve the many accelerator physics and technology issues of this type of machine. The long term goal of this work is the construction of a state-of-the-art 5 to $7 \mathrm{GeV}$ ERL-based synchrotron light source. A key element of this machine is a high brightness injector with every bunch Iled $(77 \mathrm{pC} /$ bunch at $1300 \mathrm{MHz})$. We report the design of a versatile injector for the prototype $E R L$ which also meets the requirements for a full energy light source. The injector uses a very high voltage $D C$ photoemission electron gun with a GaAs photocathode and a conventional bunching cavity. A cryomodule with ve two-cell superconducting RF cavities allows energies between 5 and $15 \mathrm{MeV}$ to be delivered, with an average beam power of $500 \mathrm{~kW}$ limited by the installed RF power. Thorough simulations, using realistic particle distributions at the photocathode, indicate this injector should provide a normalized rms transverse emittance approaching $1 \mathrm{~mm}$-mrad, and an rms longitudinal emittance of $10 \mathrm{keV}$-degrees. Operation at reduced bunch charge will provide a smaller transverse emittance, until aberrations and the time dependence of the RF elds impose limitations.
\end{abstract}

\section{INTRODUCTION}

There is considerable current interest in developing ERLs as driver accelerators for the production of synchrotron radiation. The transverse and longitudinal emittances of the full energy beam in such accelerators are not limited to the equilibrium values that prevail in similar energy storage rings, but rather are determined by the emittances produced in the injector and any growth during subsequent acceleration and transport. In principle, an ERLbased light source should deliver high $\mathrm{X}$-ray beams with beam brightness much higher, and pexlse durations far shorter than those available from storage rings.

Many accelerator physics and technology issues must be quantitatively understood before the construction of a full scale ERL light source can be responsibly proposed. To address and resolve these issues, Cornell, in collaboration with Jefferson $L a b$, has proposed the construction of a low energy, full current ERL prototype [1]. The injector is a key element of this small accelerator. It must deliver a high average current beam with very small transverse and longi-

\footnotetext{
${ }^{\circledR}$ Work supported by Cornell University

y bazarov@ cornell.edu
}

tudinal emittances, at a suf ciently high energy that space charge effects are under control. Since our proposal was written, we have developed a complete design for this injector. The performance of this design has been modeled in detail. The code ASTRA [2] was used to model the beam propagation from the photocathode through the injector cryomodule. Since bend magnets are not included in ASTRA, PARMELA [3] was used to model through the magnets that merge the injector beam with the full energy recirculated beam at the main linac entrance. Coherent synchrotron radiation (CSR) effects in the merger magnets were modeled with el egant [4]. We present here the detailed injector design, and various simulation results. These indicate that this injector design will deliver a $100 \mathrm{~mA}$ average current beam with very low transverse and longitudinal emittances. The emittance is signi cantly improved at reduced bunch charge.

\section{INJECTOR DESIGN}

The ERL will use the established TESLA SRF technology at $1300 \mathrm{MHz} .100 \mathrm{~mA}$ average current corresponds to $77 \mathrm{pC} /$ /bunch. We will use a DC photoemission gun operating at 500 to $750 \mathrm{kV}$ to deliver this current. The cathode electrode has a $20^{ \pm}$cone angle for transverse focusing. A negative electron af nity GaAs photocathode offers high quantum ef ciency and a low thermal emittance. The cathode is illuminated by a $780 \mathrm{~nm}$, harmonically mode-locked Ti:sapphire laser providing a $1300 \mathrm{MHz}$ train of » $45 \mathrm{ps}$ FWHM duration pulses [5]. The optical beam at the photocathode will be shaped to a near- transverse pro le by refractive optics [6], and will bttopa Gaussian longitudinal pro le.

The center of the rst solenoid is located $\gg 29 \mathrm{~cm}$ downstream of the photocathode, after an RF shielded gate valve. A single cell buncher cavity, adapted from the PEP-II cell design [7], is used for conventional drift bunching. The center of this cell is $50 \mathrm{~cm}$ from the center of the rst solenoid. A second solenoid follows the buncher cavity.

Acceleration to the full injector energy is provided by a cryomodule containing ve two-cell SRF cavities [8]. The center of the rst cavity is $1 \mathrm{~m}$ downstream from the center of the buncher cavity. These two-cell cavities were carefully designed to assure that harmful dipole higher-order modes propagate through the beam pipe to ferrite HOM loads [9]. B eam energy is not recovered in the injector. With an energy gain of $5 \mathrm{MeV}$ in the injector cryomodule, each cavity delivers $100 \mathrm{~kW}$ of average beam power. Each cavity has two coaxial couplers, to minimize the coupler kick and keep the power delivered per coupler at a con- 
servative $50 \mathrm{~kW}$. The couplers are variable, allowing the external $\mathrm{Q}$ to be changed. This feature allows operation at higher injector energy and reduced average current, subject only to the limitation imposed by the $500 \mathrm{~kW}$ of installed injector RF power. We will explore injector performance over an energy range of 5 to $15 \mathrm{MeV}$.

Three sector dipoles with equal bend angles merge the injected beam with the full energy single pass recirculated beam. We have calculated the effects of space charge and CSR through these merger magnets, to completely characterize the injector beam at the entrance to the main linac. Fig. 1 gives a schematic view of the full injector.

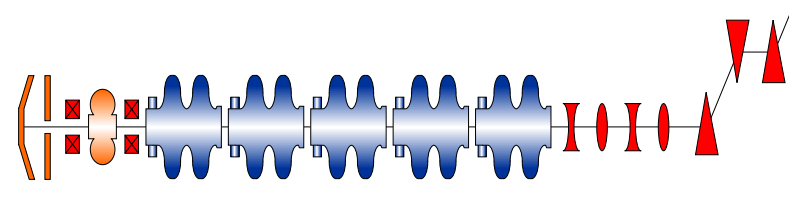

Figure 1: Injector schematic.

\section{PARAMETERS AND MODELING}

We have explored a broad range of operating parameters for the injector elements, but have not yet done a final optimization. Rather, we have sought to demonstrate a modeled performance significantly better than required for the proposed ERL, as a solid proof-of-principle that our performance requirements can be met.

Initial ASTRA modeling was done with a small number of macroparticles, and a "tophat" transverse distribution and zero thermal emittance at the cathode, to quickly

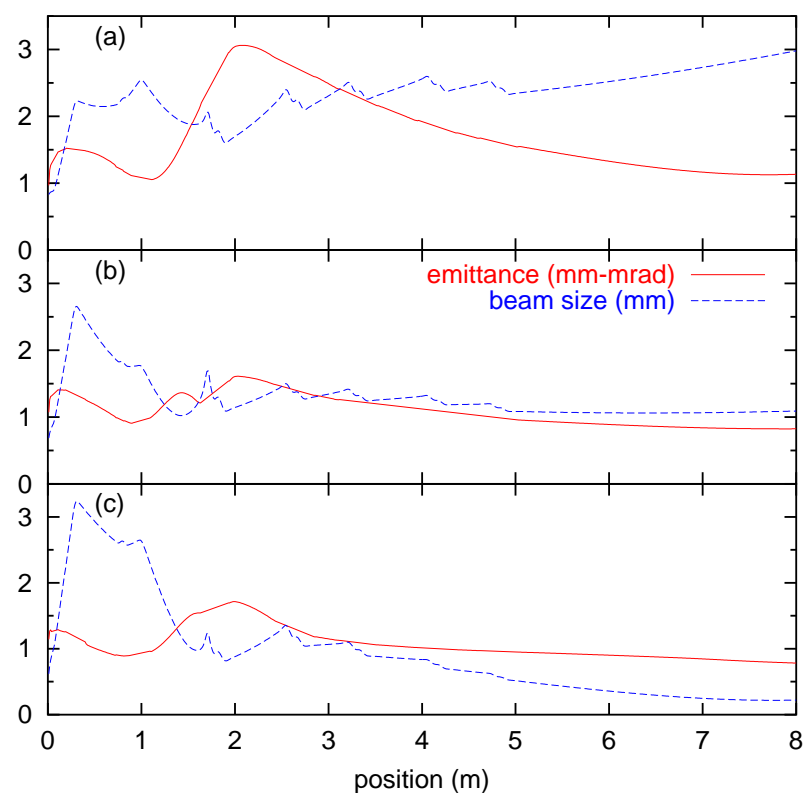

Figure 2: Rms normalized emittance and rms beam size for $77 \mathrm{pC} /$ bunch for different kinetic energies at the end of the injector: a) $6 \mathrm{MeV}$, b) $11 \mathrm{MeV}$, and c) $15 \mathrm{MeV}$

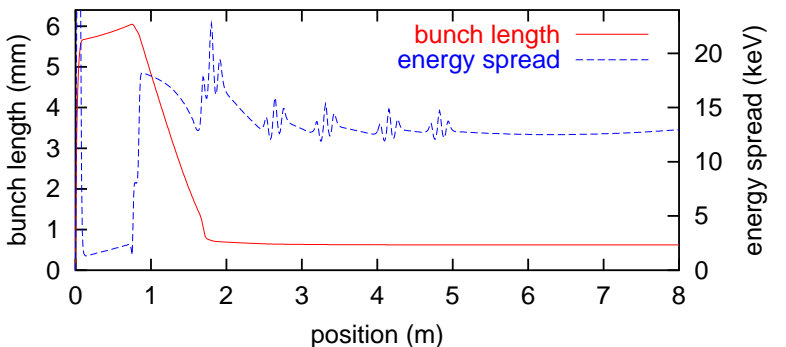

Figure 3: Rms bunch length and rms energy spread in the injector for $77 \mathrm{pC} /$ bunch, $11 \mathrm{MeV}$ final energy.

determine the best value ranges for the fields of the two solenoids and the buncher. Modeled fields were used for the gun, solenoids, and buncher and accelerator cavities.

Following the survey runs, detailed ASTRA solutions were developed at several different injector energies, using 5000 macroparticles. For these cases, the Fermi-Dirac distribution of Ref. 6 with a $\beta$ value of 15 was used for the transverse distribution at the cathode. The thermal emittance due to an effective cathode temperature of $35 \mathrm{meV}$, as measured for GaAs photocathodes, was used in all cases [10].

ASTRA modeled beam from the photocathode to $3 \mathrm{~m}$ after the cryomodule. As the superconducting cavity energy gain increases, the RF focusing in the first cavity also increases. To obtain solutions with reasonable behavior of the beam envelope, the illuminated spot size at the cathode was decreased as the energy was increased. We found well-behaved solutions from 6 to $15 \mathrm{MeV}$, with normalized transverse emittances below about $1 \mathrm{~mm}$-mrad and longitudinal emittances about $10 \mathrm{keV}$-degrees. Three such solutions are shown in Fig. 2. Typical bunch length and energy spread in the injector are shown in Fig. 3.

Emittance growth due to space charge through the merger magnets was modeled with PARMELA. The Twiss

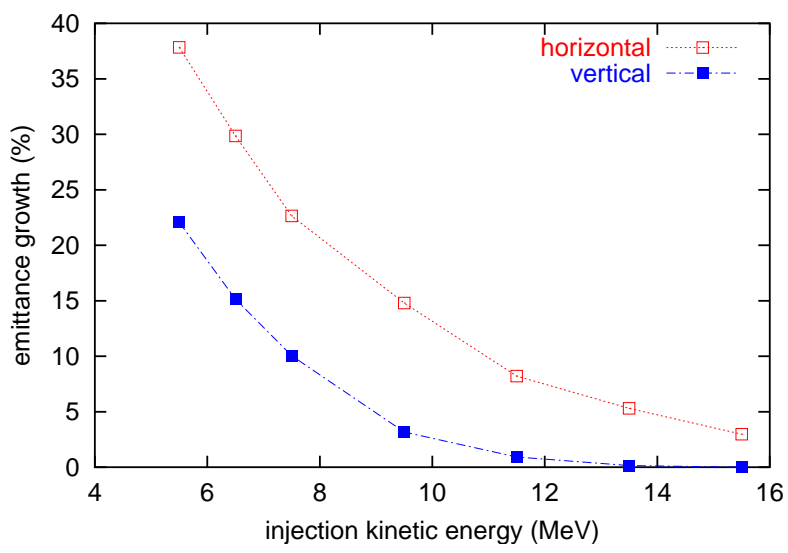

Figure 4: Emittance growth in the merger due to space charge for different injection energies. Initial rms normalized emittance is taken to be $1 \mathrm{~mm}$-mrad. 
parameters at the entrance to the merger were selected to obtain reasonable beam envelopes in the main linac, where the initial RF focusing is very strong due to the very high $(20 \mathrm{MV} / \mathrm{m})$ accelerating gradient, particularly at the lowest injector energy. The results are given in Fig. 4. Reducing the gradient in the first main linac cavity, or operating at higher injection energies will reduce the emittance growth through the merger system.

CSR effects through the merger magnets were modeled with elegant for a $15 \mathrm{MeV}, 0.6 \mathrm{~mm}$ long bunch as a function of the bend angle of the merger dipoles. No shielding effects were included. The results are shown in Fig. 5. We will use a $15^{\circ}$ bend angle for the merger magnets. The emittance growth depends strongly on the bunch length at the entrance of the merger system. The bunch length for these simulations, as determined from the previous ASTRA runs, varied between 2 and 3 ps. The optimum bunch length for a full scale ERL has not yet been determined. The simulations here apply to the case where the synchrotron radiation critical wavelength is much shorter than the bunch length. Some reduction in the effect is possible at the lowest injector energy, where the critical wavelength is the longest.

Operation of the full energy ERL with reduced emittance, obtained by reducing the bunch charge, will be important for some applications. By reducing the bunch charge a factor of ten, we have obtained a significantly smaller final emittance, as shown in Fig. 6. While the ther-
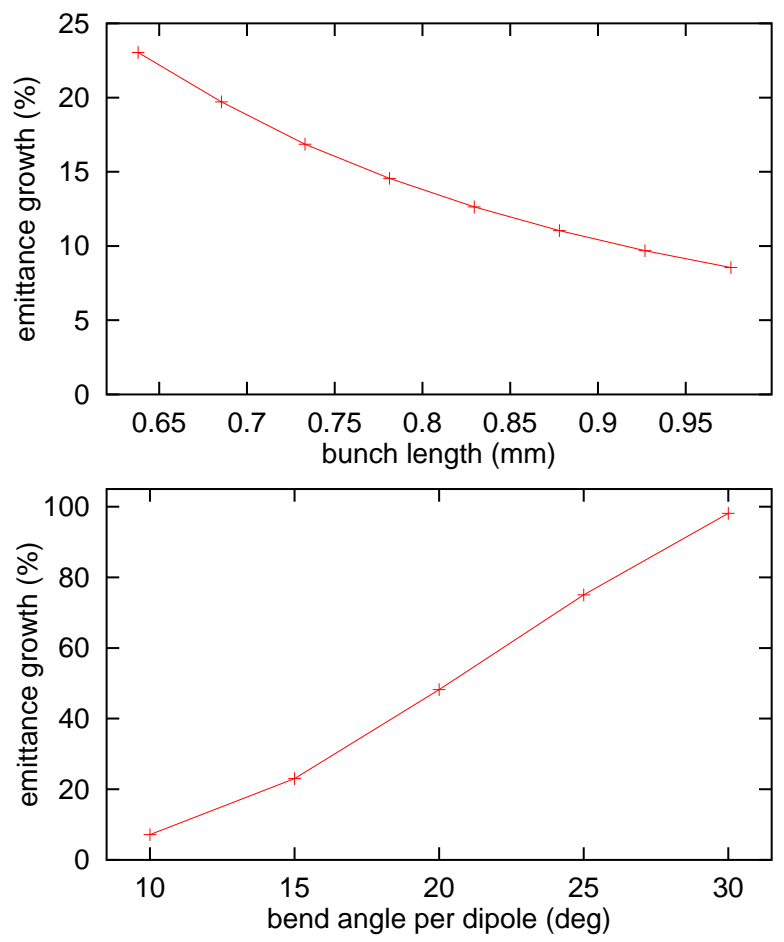

Figure 5: Emittance growth in the merger due to CSR for 77-pC bunch as a function of rms bunch length (top) and dipole bend angle for $0.6-\mathrm{mm}$ bunch (bottom). Initial rms normalized emittance is taken to be $1 \mathrm{~mm}$-mrad.

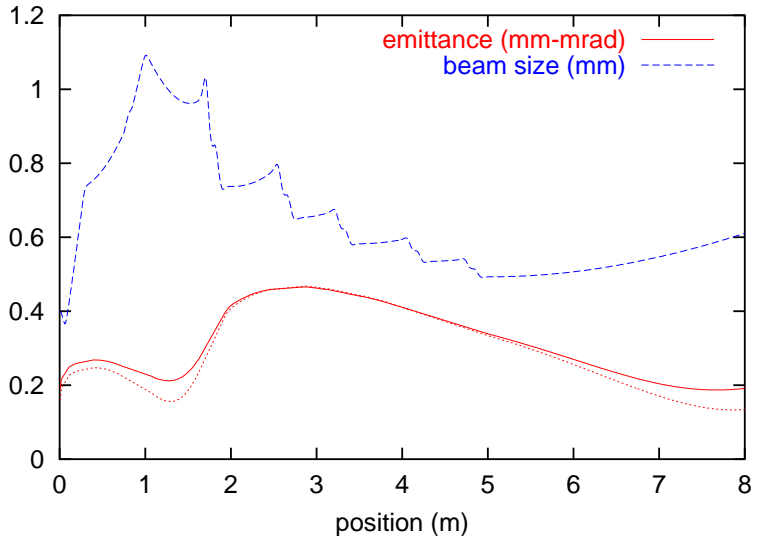

Figure 6: Rms normalized emittance and rms beam size for $8 \mathrm{pC} / \mathrm{bunch}$. Dotted line shows emittance in case of zero thermal emittance at the photocathode. Kinetic energy at the end of the injector is $6 \mathrm{MeV}$.

mal emittance of the cathode is a noticeable contribution in this case, space charge remains the major source of emittance growth. We plan to conduct further optimizations for the low emittance, reduced bunch charge case.

\section{CONCLUSIONS}

Based on the simulations we have conducted to date, we are confident that our ERL injector design will deliver a beam with rms normalized transverse and rms longitudinal emittances of about $1 \mathrm{~mm}$-mrad and $10 \mathrm{keV}$-degrees. The strong RF focusing in the initial cavity of the injector, and again in the first cavity of the main linac, complicates finding the optimum parameters. We plan to conduct a more complete exploration of all parameters using computer-aided optimization routines.

\section{REFERENCES}

[1] S.M. Gruner, M. Tigner (eds.), CHESS TM 01-003, available at http://erl.chess.cornell.edu/papers/ERL_Study.pdf

[2] K. Flöttmann, ASTRA User Manual, September 18, 2000.

[3] L. Young, PARMELA Reference Manual, LA-UR96-1835, January 8, 2000.

[4] M. Borland, elegant: A Flexible SDDS-Compliant Code for Accelerator Simulation, APS LS-287, September 2000.

[5] C. Hovater and M. Poelker, NIM A 418, 280 (1998).

[6] J. Hoffnagle and C. Jefferson, Appl. Opt. 39, 5488 (2000).

[7] V. M. Vescherevich and S. Belomestnykh, Buncher Cavity for ERL, Proceedings of PAC'03.

[8] H. Padamsee et al., Overview of the Cornell ERL Injector Cryomodule, Proceedings of PAC'03.

[9] V. Shemelin et al., Dipole-Mode-Free and Kick-Free 2-cell Cavity for the SC ERL Injector, Proceedings of PAC'03.

[10] B.M. Dunham et al., Emittance Measurements for the Illinois/CEBAF Polarized Electron Source, Proceedings of PAC'95. 